\title{
Difficulties Caused by Magnetic After-effect during Identification of the Preisach Hysteresis Model Weighting Function
}

\author{
M. NOVÁK \\ Institute of Mechatronics and Computer Engineering, Technical University of Liberec, \\ Studentská 2, CZ 46117 Liberec, The Czech Republic
}

\begin{abstract}
The time dependence of the magnetic hysteresis caused by thermal activation over an energy barrier is called after-effect, magnetic viscosity, or magnetic relaxation. Magnetic after-effect influences the hysteresis loop shape depending on an excitation filed rate of change, the sample geometry, and the state. The magnetizing loop changes, especially at rounding of peaks at steep part of the loop, severely influences process of the Preisach model weighting function identification from experimental data. This article deals with the after-effect measuring with the aim of determining limiting excitation speed.
\end{abstract}

DOI: 10.12693/APhysPolA.136.731

PACS/topics: 75.60.Lr, 75.60.Ej, 85.70.Ay

\section{Introduction}

The time dependence of a ferromagnetic material magnetization under a constant magnetic exciting field is called magnetic after-effect. This phenomenon occurs due to thermal activation of the irreversible magnetization processes [1]. It is the result of approaching the thermodynamic equilibrium with a minimum of free energy in the material where the energy distribution is complicated. Magnetic domain walls can be trapped in local minimums of energy for a long time until they are excited by thermal fluctuations and they overcome the energy barrier. The material exhibits thermally activated Barkhausen jumps and it moves step by step towards a low energy state. The rate of this relaxation depends on the distribution of energy barriers and temperature.

The overall magnetization is composed of a reversible and an irreversible component $M(t)=M_{\text {rev }}(t)+M_{\text {irr }}(t)$. Although, the distribution of energy barriers can be a general function the most of the ferromagnetic materials exhibit logarithmic decay of magnetization over the time

$$
M_{\text {irr }}(t)=M_{0}-S \ln \left(t / t_{0}\right),
$$

where $t$ is the time since changing the excitation field, $M_{0}=M_{\text {irr }}\left(t=t_{0}\right)$, and $S$ is the relaxation coefficient $[1,2]$. The magnetic after-effect can be observed for a change of the field and it requires much longer time to attain a new steady state compared to the much faster eddy current effect. A similar differential model of an after-effect is presented in [3].

The first order reversal curves (FORC) method is one of the methods used for identification of the weighting

e-mail: miroslav.novak@tul.cz function (WF) of the Preisach model of hysteresis $(\mathrm{PMH})[4,5]$. It is based on measuring a set of hysteresis loops. Each loop starts from its negative saturation level. Consequent loops gradually increase the maximum of the exciting field strength $H_{U}$. The descending branch of the loop, the first order reversal part, is inserted into a 2D matrix. The magnetization of the descending branch $M\left(H_{D}\right)$ creates rows of the matrices that are placed into columns in accordance with $H_{U}$. Such surface is called the Everett function, see Fig. 1. The second partial derivative of the Everett function results in the WF of PMH:

$$
w\left(H_{U}, H_{D}\right)=-\frac{1}{2} \frac{\partial^{2} M\left(H_{U}, H_{D}\right)}{\partial H_{U} \partial H_{D}} .
$$

The rounding of the magnetizing loops caused by the after-effect takes place especially at the area of their steep part where an irreversible magnetization process dominates. The magnetization $M(H)$, due to the aftereffect time delay, still grows while the exciting field strength $H$ already starts to decline resulting in rounding of the loop tip. A key problem is the stochastic fundamental of the after-effect that causes random curvature. See an example of repeated measurements under the same conditions in Fig. 2. The stochastic behavior of the after-effect causes variation of the loop trajectory and different value of reached maximal flux density $B_{\max }$.

Such rounded tops of the magnetizing loop lead to non-physical negative angular coefficients at the edge of the Everett function close to the hypotenuse of the Preisach triangle. Random variable value $B_{\max }$ causes severe problem with the second derivative of the Everett function leading to a deterioration of WF and of the simulated hysteresis loops.

The only correct way to suppress the loop tip rounding is to slow down frequency of the excitation field during measuring. On the other hand, the extra low frequency 


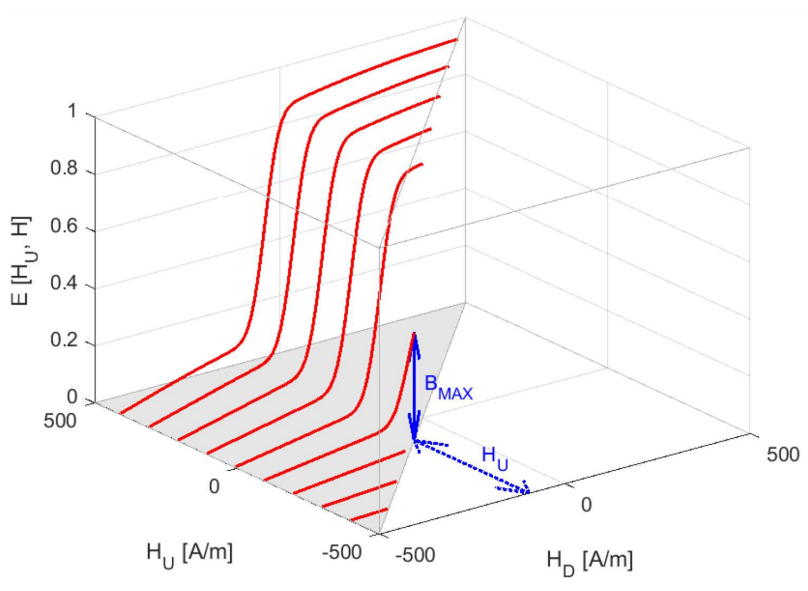

Fig. 1. Formation of the Everett surface from descending branches of magnetizing loops.

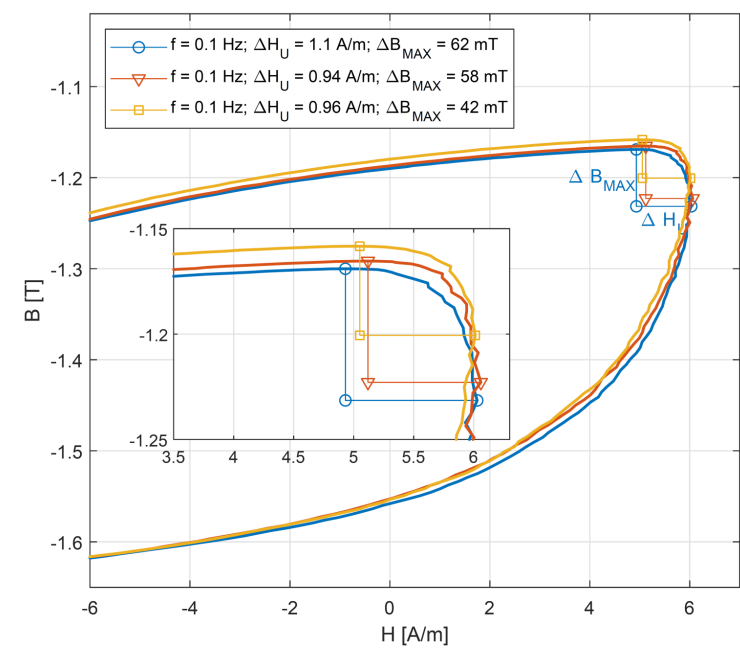

Fig. 2. Rounded tips of FORC loops caused by aftereffect, material M165-30S, longitude direction.

measurement brings other kind of problems, e.g. the offset drift during the magnetic flux integration, the offset drift of exciter, and so on. The aim of this article is to determine the time constant of the magnetic after-effect and the proper frequency setting of the FORC measurement.

\section{Magnetic after-effect measurement}

There are several methods to measure the magnetic after-effect. Magnetically hard materials are usually measured using a vibrating magnetometer while the time response of the magnetization is generated from a jump of the field strength most often to the coercive force value $H_{c}[2,6]$. Other approach is to measure time dependence of the inductance or susceptibility using a small signal after the bulk field strength change $[1,7]$. Other authors analyze dynamics hysteresis loops and determine the ratio of the eddy current and the after-effect [8].
Our primary concern is a quantitative analysis of the after-effect alongside the magnetizing loop. Therefore the magnetization response in time $M(H, t)$ after small jumps of the field strength is measured. The magnitude of jumps is variable and is chosen to keep the constant move of magnetization $\Delta M=$ const. The idea is to achieve the same volume changes in the domain wall shifts. For this reason, the major hysteresis loop $B(H)$ is measured at a frequency of $0.1 \mathrm{~Hz}$ before the after-effect measurement itself. This major loop data determine the jumps of the excitation current so that the change in the flux density in one jump is constant $\Delta B=50 \mathrm{mT}$.

The setup used for the after-effect measurement was identical with the FORC measurement, where the current excitation is required to accurately define the field strength $H$, see Fig. 3. The excitation signal (jumps) is digitally generated by the DAC as the distribution function of the normal distribution

$$
F(x)=\int_{-\infty}^{x} \frac{1}{\sigma \sqrt{2 \pi}} \exp \left(-\frac{(t-\mu)^{2}}{2 \sigma^{2}}\right),
$$

where the center of the distribution is $\mu=0.1 \mathrm{~s}$ and the standard deviation is $\sigma=0.015$. The smooth transition function suppresses the influence of eddy currents and reduces the distortion of the signal by the power amplifier slew rate, see $H$ curve in the inset of Fig. 3 .

The power amplifier OPA549 is connected as an inverting voltage-to-current converter to provide current for the excitation coil of the standard Epstein frame (IEC 60404-2). The field strength $H$ is determined indirectly and is calculated from the measured exciting current.

The closed magnetic circuit is formed from three layers of steel sheet strips of a material sample. The grain oriented steel (GOSS) with different grade and orientation to the rolling direction was tested (M165-30S, M097-30N).

The secondary voltage response was captured by $24 \mathrm{bit}$ $\mathrm{ADC}$ at $50 \mathrm{kSPS}$ for $10 \mathrm{~s}$ after each jump. The magnetic flux is calculated from the secondary voltage using induction law, where initial flux is determined from saturation starting point [9]. The corresponding magnetic flux density transients are shown in Fig. 4b for different selected positions on the magnetization loop (their positions are marked in the left hand graph in Fig. 4a). The flux density time constant $\tau=(1-1 / \mathrm{e}) \Delta B$ varies from a fraction of milliseconds at the saturation region up to more than two seconds in the steep part of the loop and again shortens when approaching the positive saturation. The markers placed in the graph are located at the moment of the relaxation time $\tau$.

The theory of the after-effect expects logarithmical response (1). Measurements revealed that this theoretical assumption is valid in areas where the time constants are small, i.e. at the saturation and at the beginning of the knee regions of the hysteresis loop, but at the steep part of the loop the response is irregular with a very long full stabilization time. 

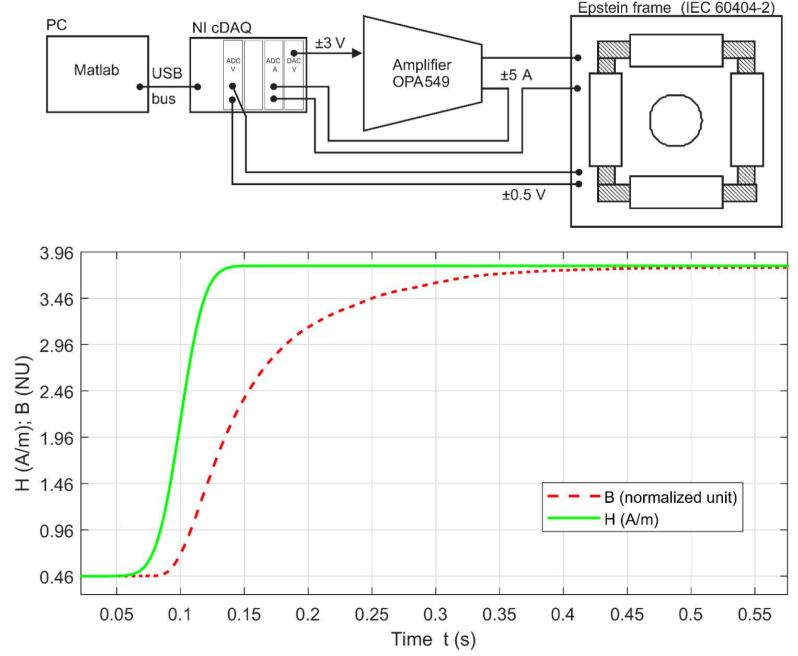

Fig. 3. Measuring setup.
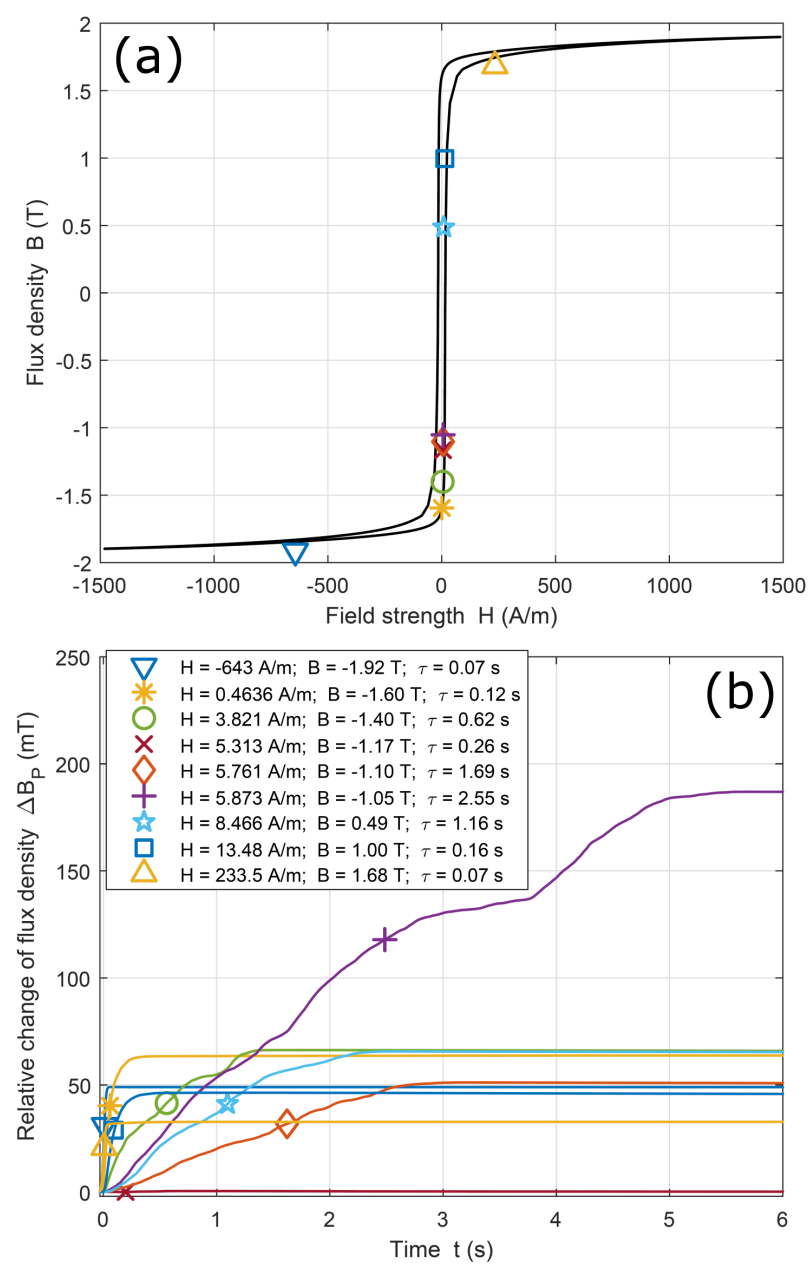

Fig. 4. Time response of flux density to small jumps of field strength (b) at different sections of the magnetizing loop (a), sample M165-30S longitude direction, few selected steps.

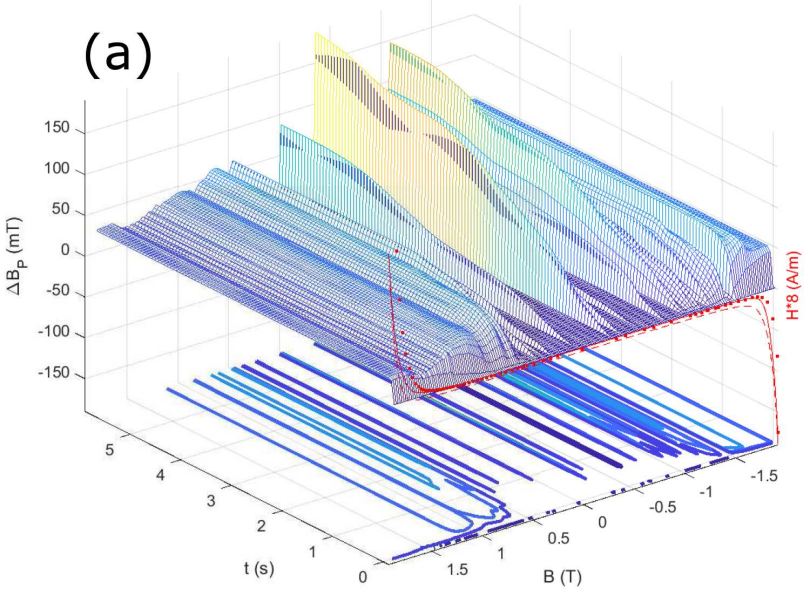

(b)

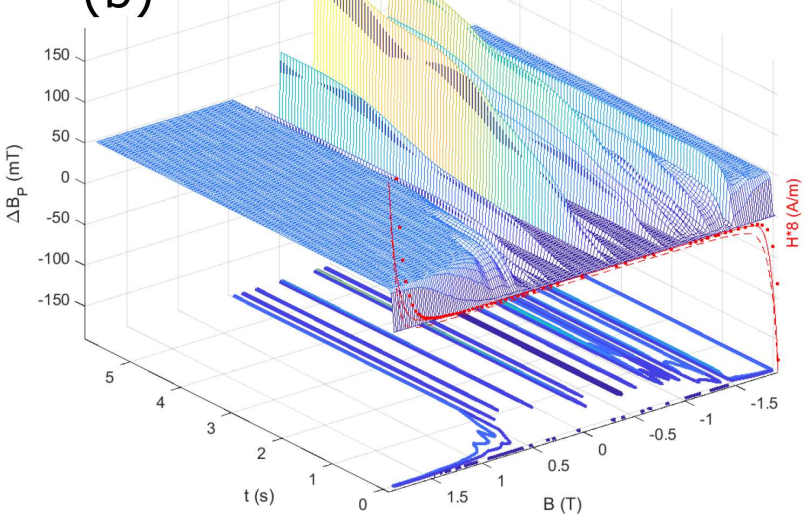

Fig. 5. Time response of flux density to small jumps of field strength for different points at the magnetizing loop, sample M165-30S longitude direction: (a) raw data, (b) normalized.

The release of energy barriers by the thermal activation is quite evident in Fig. $4 \mathrm{~b}$ waveforms $(\bigcirc,+$, and $\diamond)$. The curves are composed of several interlacing logarithmic sections. Places where unblocking of energy barriers occurs are random. In some jumps, the energy supplied by the driver to the sample is insufficient to overcome the domain wall motion energy barrier to change the magnetization. In this case, the flux density response is almost zero (see $\times$ mark in Fig. $4 \mathrm{~b}$ ).

The dataset for the ascending branch of the majority loop is presented as a 3D plot in Fig. 5a. The major hysteresis loop with marked points of the individual measured jumps is shown as red loop on the front side of the graph. The signal at the end of each jump should end at roughly $50 \mathrm{mT}$. Deviations occur due to difference of the major loop measured at $0.1 \mathrm{~Hz}$ and loop trajectory during the step measurement and mainly because of the stochastic nature of the irreversible magnetization. The normalization of the near values was performed in the graphs in Fig. 5b to improve the clarity of the time response development. 

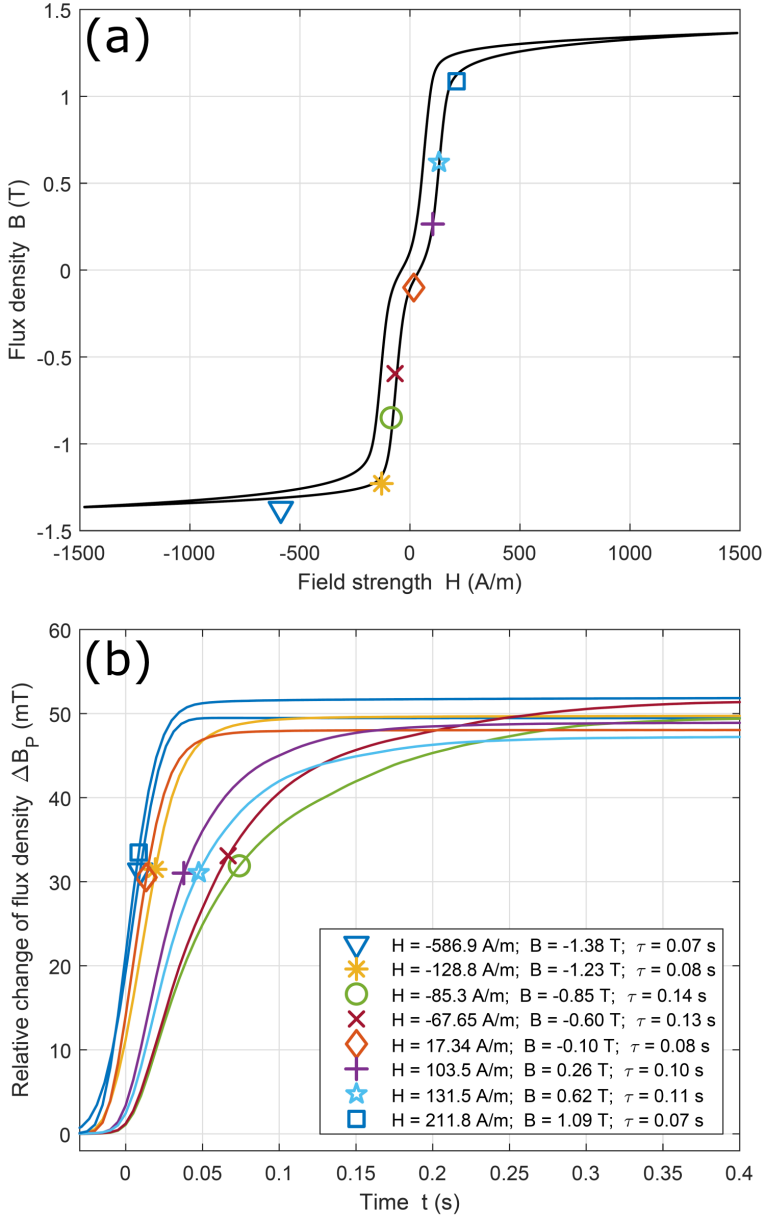

Fig. 6. Time response of flux density to small jumps of field strength (b) for different points at the magnetizing loop (a), sample M165-30S transverse direction, few selected steps.

As noted above, it is clear that in the saturation region, the time constant of the after-effect is negligible. In the knee, the time constant increases steadily and has a logarithmic course. The steep region of the major loop contains a number of very different complex waveforms with long time constants interleaved by spaces where, although a jump of the field strength $H$ was generated, there was no response to the flux density $\Delta B$. Typically, such omitted jumps are followed by a measurement where the final flux density is the sum of the omitted jumps.

The same material M165-30S with the transverse orientation of the sample towards the direction of the easy magnetization is presented in Figs. 6 and 7. Magnetizing takes place for the direction chosen at $90^{\circ}$ to the easy axis magnetization, the major loop is not so steep and has typical delay around $H=0$. There are only jumps with a time constant of up to $0.2 \mathrm{~s}$. There are absolutely no jumps without the flux density response.

Another example is material M097-30N which has not undergone annealing after the mechanical treatment. This leads to a deterioration of magnetic properties,
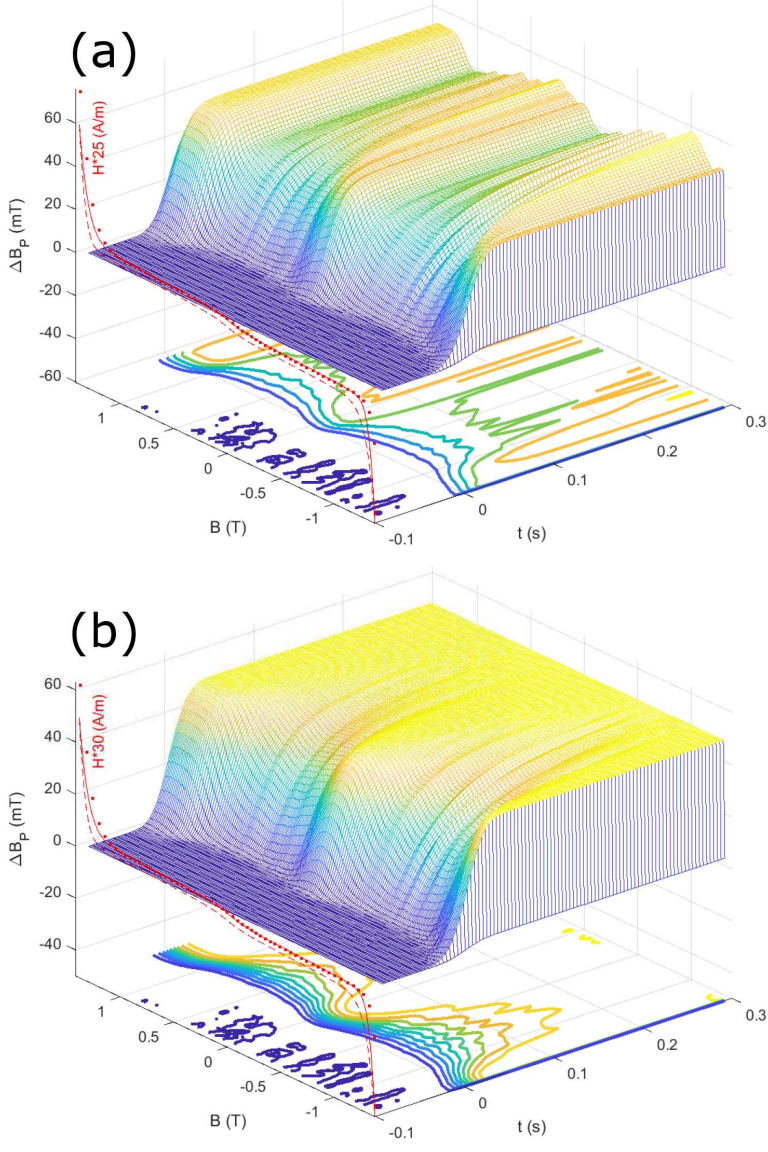

Fig. 7. Time response of flux density to small jumps of field strength for different points at the magnetizing loop, sample M165-30S transverse direction: (a) raw data, (b) normalized.

losses, and also to an enlarged area of the loop knee, see Figs. 8, 9. The results show all the phenomena as the previous sample M165-30S measured in the longitudinal direction but their magnitude and the area where they are found on the major loop are different.

\section{Discussion}

The change of magnetization caused by after-effect is theoretically logarithmic one (1). The essence of the process is stochastic - it is based on overcoming energy barriers of the domain walls motion caused by thermal activation. The distribution of the barriers (vacancies, inclusions, and mainly dislocations) in the crystal is a random function. If we neglect diffuse migration and changes of the mechanical stress, the obstacles are firmly placed. There are obstacles in the material with a significantly higher energy needed to overcome them or overcome clusters of obstacles, so the measured magnetization response in time is not purely logarithmic but contains a curve composed of multiple logarithmic functions, see Figs. 4-9. 

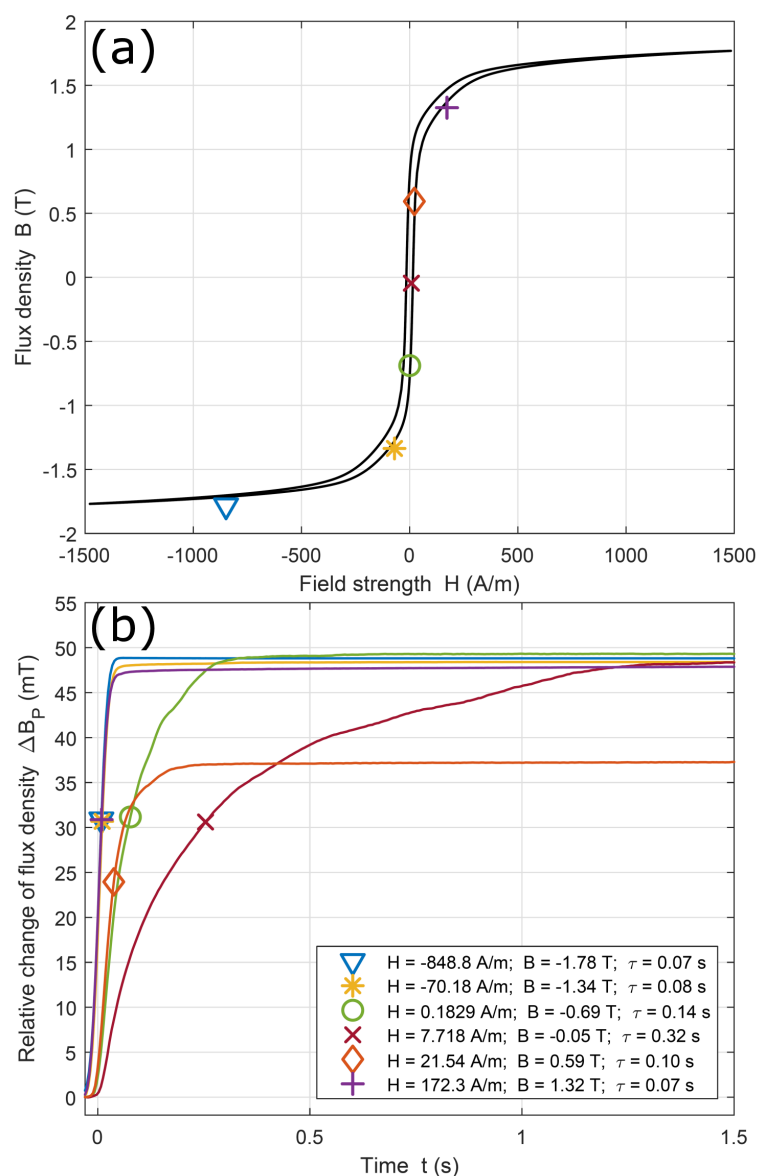

Fig. 8. Time response of flux density to small jumps of field strength (b) at different sections of the magnetizing loop (a), sample M097-30N longitude direction, few selected steps.

The magnetization jumps observable after overcoming energy barriers are repeatedly measured at the same locations, i.e., at the same field strengths $H$, see three independent loops measured with the same conditions in Fig. 10. On the other hand, the randomness of the thermal activation causes the given jumps shift toward lower field strengths of $H$, leading to an increase of the maximal flux density reached at the loop tip, see Fig. 2.

The magnetization achieves the reversible magnetization $M_{\text {rev }}$ (anti-hysteresis curves) in an infinite time (1), see Fig. 11 and [10]. Some obstacle stops domains wall motion sooner and they do not move further regarding to the measuring accuracy. This was verified by determining the derivative $\mathrm{d} M / \mathrm{d} t$ at the end of each measurement interval of each jump.

The rounded tips of the loops can be suppressed by reducing the frequency. The after-effect reduces the coercive force $H_{c}$ due to removal of the irreversible magnetization component $M_{\text {irr }}$, see Fig. 12. The implementation of the PMH with the WF obtained by such measurements must include the after-effect model to compensate for the $H_{c}$ decrease [11].
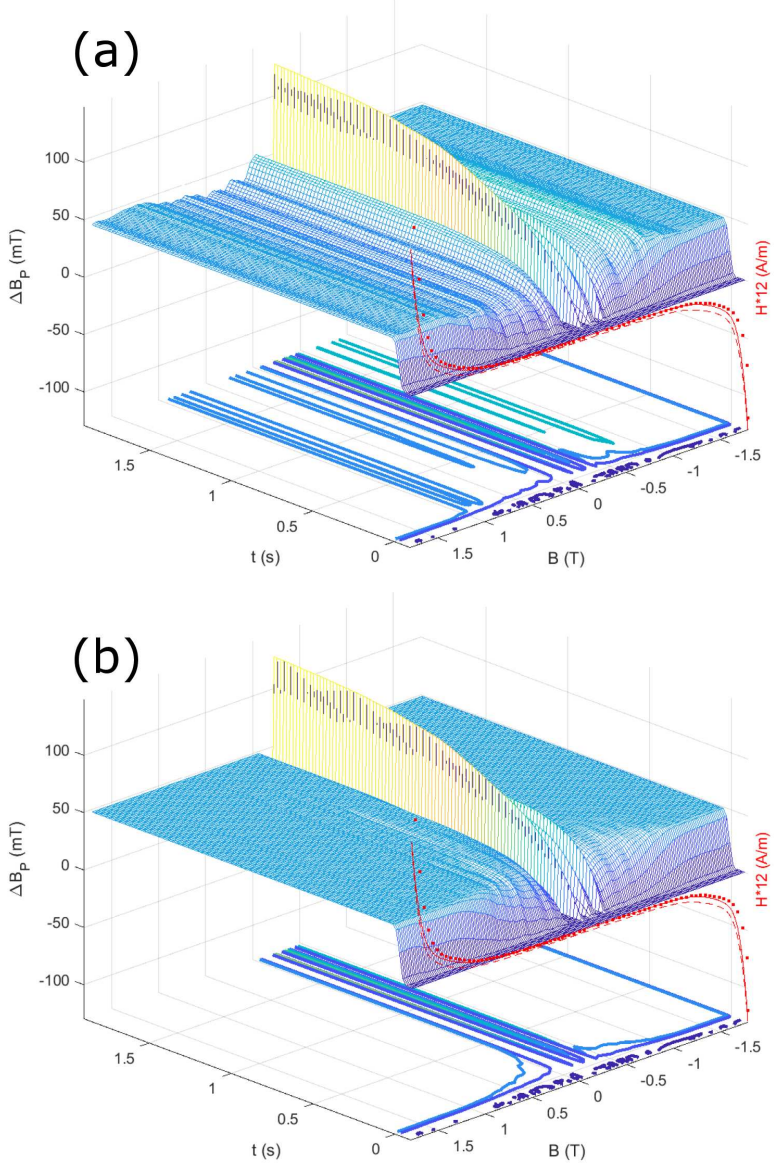

Fig. 9. Time response of flux density to small jumps of field strength for different points at the magnetizing loop, sample M097-30N transverse direction: (a) raw data, (b) normalized.

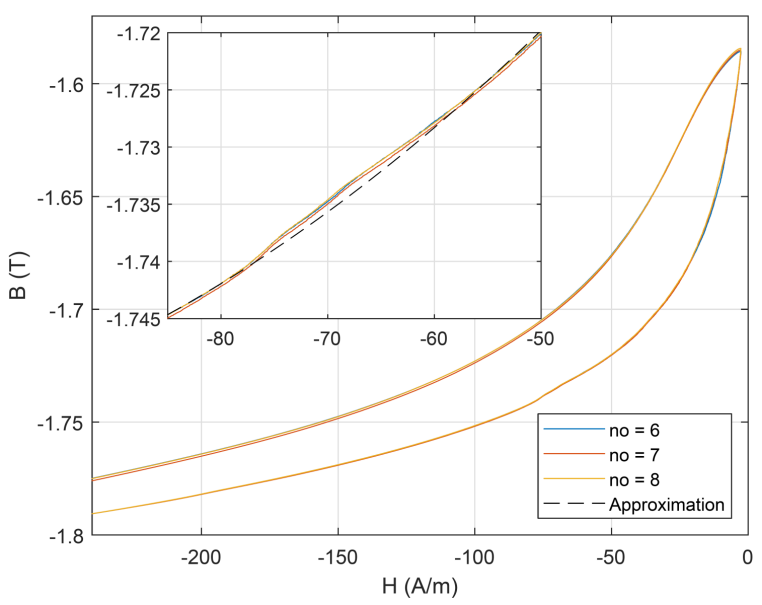

Fig. 10. Reproducibility of the energy barrier overcoming, sample M165-30S longitude direction, $f=0.01 \mathrm{~Hz}$.

Measuring FORC with a sinusoidal waveform is not correct from the viewpoint of the after-effect because the slope of the sinusoidal function is variable. The steep part of the FORC loop is measured with a different slope 


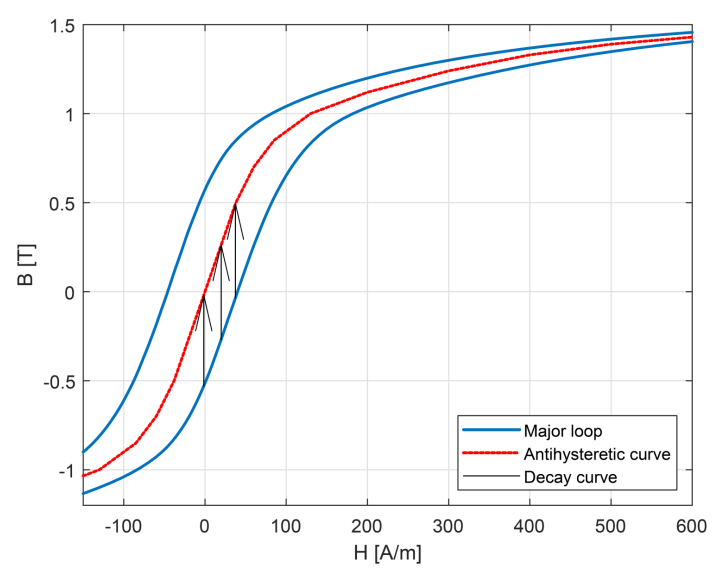

Fig. 11. Magnetization decay due to after-effect.

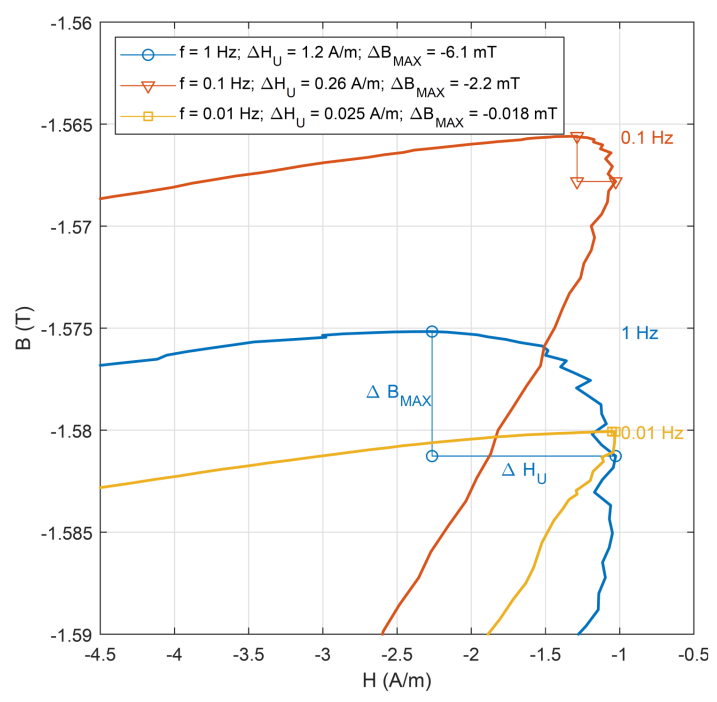

Fig. 12. Rounded tips of FORC loops in dependence on exciting frequency, sample M165-30S longitude direction.

of $\mathrm{d} H / \mathrm{d} t$ for cycles with different $H_{U}$. Stable conditions ensure measuring by a triangular excitation waveform. Instead, the triangular signal creates a problem at the tip of the loop where the $\mathrm{d} H / \mathrm{d} t$ sign changes. The use of a trapezoidal excitation is potentially suitable with persistence at $\underline{H}_{U}$ value with smooth transitions.

The Everett function obtained by the FORC measurement will always necessarily contains random shifts of the separate curves caused by the after-effect. This transverse roughness in direction of $H_{U}$ cannot be eliminated by reducing the measuring frequency. Repeated measurements of FORC curves for the same value of $H_{U}$ with subsequent statistical evaluation is a possible solution.

\section{Conclusion}

We have shown that the after-effect is easily measurable under normal conditions. The presented method quantitatively determines its influence over the entire magnetization range. The influence of the after-effect is particularly important in the steep part of the magnetization loop. It is possible to determine the time constant and to set the optimal frequency of the FORC measurement. This will suppress the rounded tips of the loops and the erroneous values at the edge of the Everett function.

The roughness of the Everett function is a natural consequence of the random nature of the after-effect. It is not necessary to remove this roughness for the PMH implementation but it is necessary to measure the data for WF correctly. This means a repeated measurements and a statistical evaluation.

It will also be necessary to verify the invariance of the sample weight. We expect random jumps to be subject to the law of large numbers that will be suppressed by larger volume of material.

\section{Acknowledgments}

The result of the work was obtained by through the financial support of the Ministry of Education, Youth and Sports of the Czech Republic, and the European Union (European Structural and Investment Funds - Operational Program Research, Development and Education) in the frames of the project "Modular platform for autonomous chassis of specialized electric vehicles for freight and equipment transportation", Reg. No. CZ.02.1.01/0.0/0.0/16_025/0007293.

\section{References}

[1] N. Abeywickrama, Y.V. Serdyuk, S.M. Gubanski, IEEE Trans. Power Deliver. 23, 3 (2008).

[2] C. Serletis, K.G. Efthimiadis, J. Magn. Magn. Mater. 324, 16 (2012).

[3] S.E. Zirka, Y.I. Moroz, P. Marketos, A.J. Moses, IEEE Trans. Magn. 42, 9 (2006).

[4] G. Bertotti, I. Mayergoyz, The Science of Hysteresis Elsevier, 2005.

[5] M. Novak, J. Eichler, M. Kosek, Appl. Math. Comput. 319 (2018).

[6] E. Cardelli, E. Della Torre, A. Faba, M. Ovichi, IEEE Trans. Magn. 49, 5 (2013).

[7] B. Minov, M.J. Konstantinović, L. Dupré, Przegląd Elektrotechniczny 87, 9b (2011).

[8] A.P.S. Baghel, S.K. Shekhawat, S.V. Kulkarni, I. Samajdar, Physica B 448, (2014).

[9] B. Tellini, R. Giannetti, S. Lizon-Martinez, M. Marracci, IEEE Trans. Instrum. Meas. 58, 8 (2009).

[10] S. Gu, W. He, M. Zhang, T. Zhuang, Y. Jin, H. ElBidweihy, Y. Mao, J.H. Dickerson, M.J. Wagner, E.D. Torre, L.H. Bennett, Sci. Rep.-UK 4, 6267 (2014).

[11] O. Alejos, E.D. Torre, Physica B 306, (2001). 